\title{
BMJ Open Use of dating sites and applications by women and their risk of sexually transmitted infections: a systematic review and meta-analysis protocol
}

\author{
Janice França Queiroz, ${ }^{1}$ Kleyton Santos Medeiros, ${ }^{1}$ \\ Ayane Cristine Alves Sarmento, ${ }^{1}$ Michelly Nóbrega Monteiro, ${ }^{1}$ \\ Ricardo Ney Cobucci, ${ }^{2}$ Beatriz Stransky, ${ }^{3}$ Ana Katherine Gonçalves (D) 1,4
}

To cite: Queiroz JF, Medeiros KS, Sarmento ACA, et al. Use of dating sites and applications by women and their risk of sexually transmitted infections: a systematic review and metaanalysis protocol. BMJ Open 2020;10:e038738. doi:10.1136/ bmjopen-2020-038738

- Prepublication history for this paper is available online. To view these files, please visit the journal online (http://dx.doi. org/10.1136/bmjopen-2020038738).

Received 21 March 2020 Revised 05 0ctober 2020 Accepted 17 October 2020

D) Check for updates

(c) Author(s) (or their employer(s)) 2020. Re-use permitted under CC BY-NC. No commercial re-use. See rights and permissions. Published by BMJ.

For numbered affiliations see end of article.

\section{Correspondence to} Dr Ana Katherine Gonçalves; anakatherine_ufrnet@yahoo. com.br

\section{ABSTRACT}

Introduction The use of social networks has been increasing worldwide. Mobile websites and applications (apps) allow people to network more quickly and have more partners for sex. This can facilitate risky sexual behaviours, such as having multiple partners and unprotected sex, which can lead to a higher incidence of sexually transmitted infections. This systematic review/ meta-analysis will assess the effects of the use of dating sites and apps by women on their level of engagement in risky sexual behaviours and their incidence of sexually transmitted infections.

Methods and analysis The Cochrane Central Controlled Trials Registry, ClinicalTrials.gov, MEDLINE, Embase, SciELO, Web of Science, Scopus and Cumulative Index to Nursing \& Allied Health Literature will be searched for cross-sectional studies, clinical trials and observational studies published between January 1990 and July 2020. This systematic review and meta-analysis will include studies investigating the use of mobile apps by women, risky sexual behaviour and sexually transmitted infections. The outcome will be an increase in new cases of sexually transmitted infections and HIV among women using dating sites and apps. Three independent reviewers will select the studies and extract data from the original articles. The risk of bias will be assessed using the Cochrane risk of bias tool and Risk Of Bias in Non-randomized Studies of Interventions. Data synthesis will be performed using Review Manager software (RevMan V.5.2.3). To assess heterogeneity, we will compute the $I^{2}$ statistic. In addition, a quantitative synthesis will be carried out if the included studies are sufficiently homogeneous.

Ethics and dissemination This study will be a review of the published data, and thus ethical approval is not required. The findings of this systematic review will be published in a peer-reviewed journal.

PROSPERO registration number CRD42019120494.

\section{INTRODUCTION}

Technological advances have created virtual reality and digital media (eg, cellphones, tablets, websites and social networks), infinitely expanding access to communication and making digital reality part of the daily lives
Strengths and limitations of this study

- This systematic review and meta-analysis will combine the results of different studies that have comparable effect sizes and can be computed.

- After selecting the articles, three reviewers will independently extract the data and assess risk of bias.

- There is a possibility that the sample size will be small, and there will be a limited number of studies because few studies have focused on the use of applications and sexual encounters.

- Different types of mobile applications and dating sites can cause considerable heterogeneity, which can limit the generation of convincing conclusions.

of individuals. ${ }^{1}$ The increasing accessibility of the internet and mobile devices (eg, laptops, smartphones and tablets) has changed the way people communicate, allowing greater mobility and speed. ${ }^{2} 3$ Mobile applications (apps) with global positioning system (GPS) capabilities allow users to expand their contacts on social networks; they are able to view profiles, send messages, make friends and find partners for the purpose of sexual encounters. $^{2}$

The use of apps by people aged 18 years and over increased from $8 \%$ in 2005 to $72 \%$ in $2013 .{ }^{4}$ Recent data indicate that $23 \%-68 \%$ of people in developing countries use the internet, and among these, $77 \%$ use sites such as Facebook, Twitter, Instagram, Pinterest and others. ${ }^{5}$ The number of people who use mobile apps is expected to reach 182 million in the near future. ${ }^{6}$ Recently, the use of apps for mobile devices has increased by $90 \%$, representing $77 \%$ of the total time spent by people using digital media. ${ }^{2}$

From 2009 to 2013, an increase in the use of these apps was observed. In 2013, $40 \%$ of men who have sex with men reported that 
they used apps to search for sexual partners, and there was an increase of approximately 7 million new users of these apps. With the proliferation of dating sites and apps and their increased use, users have the opportunity to have more casual sexual encounters, resulting in unsafe sexual practices, increasing the chances of getting a sexually transmitted infection (STI) ${ }^{7}$

In a study of 20091 Australian residents, ${ }^{8} 12.09 \%$ of participants (13.52\% men and $10.65 \%$ women) reported having looked for potential partners on websites and smartphone apps, and $7.01 \%$ men and $3.77 \%$ women reported doing so in the last year. Among sexually active women, the majority were heterosexual $(96.09 \%)$ and $52.31 \%$ were between 16 and 39 years old. Women who reported having more than two sexual partners in a year were more likely to report having sex with someone they met online than those who only had one sexual partner. In addition, women who underwent tests or were diagnosed with STI reported more sexual intercourse with partners they met on social networks than those who did not have STI. ${ }^{8}$

Evidence from these studies is controversial because some results have suggested that users of dating apps may have more opportunities to perform safe sexual practices than people who do not use these apps, thus not enhancing risky sexual behaviour or the risk of subsequent infection with an STI. ${ }^{9}$ Therefore, a comprehensive study on the use of dating sites and apps and its effect on sexual health is warranted.

\section{Description of the intervention}

In recent years, advances in communication technology have offered geosocial networks equipped with GPSs (eg, Facebook and Instagram) and dating apps (eg, Tinder and Badoo) for men and women, and with these come different opportunities to meet sexual partners.

With the proliferation of dating sites and apps, their use can facilitate the search for casual sexual partners, resulting in unsafe sexual practices. ${ }^{10}$ Studies have shown that users of these apps tend to have a greater number of sexual partners, more frequent sexual encounters, more unprotected sex and a greater number of sexual partners with HIV and other STIs. This increases the risk of acquiring/transmitting HIV and STIs, compared with individuals who use different methods of finding sexual partners. ${ }^{11}$

A systematic review of the literature using articles from databases will therefore be carried out with the aim of assessing the effect of the use of dating sites and apps by women on their increase in risky sexual behaviours and incidence of STIs.

\section{Intervention mechanisms}

Websites, mobile apps and social networks for encounters that involve risky sexual behaviour and STI. ${ }^{12}$

The technology makes it easier for users to get to know more people, relate sexually and have unprotected relationships, ${ }^{9}{ }^{10}$ increasing the likelihood of subsequent infection with an STI. ${ }^{9}$

Mobile apps can influence and offer various behavioural interventions and can contain and provide access to prevention and sexual education tools for their users. ${ }^{13}$

Applications provide a new platform for delivering tailored HIV and STI prevention and care. ${ }^{14} 15$ Alhassan et $a l^{15}$ demonstrated that young adults would prefer to use mobile phone apps for STI education and prevention. The same study recommended that public health practitioners work with app developers to incorporate elements of evidence-based interventions for risk reduction and improve app inclusiveness and interactivity. ${ }^{1415}$

\section{Why it is important to perform this review}

Traditional interventions to understand the use of social networks by women and their effects on health and sexual behaviour are insubstantial and are not widely known. ${ }^{13}$

There is a widespread need for scientific and technological innovations that provide convenient information about the behavioural profile of these women. With the use of social media apps to find sexual partners, these women tend to have more partners, more casual sexual encounters and more frequent anal sex. They also more frequently have sex without a condom, which can increase their risk of contraction and transmission STI. ${ }^{7}$

According to the WHO, the prevalence of STIs remains high worldwide. Since 2016, it is estimated that there have been 376 million new infections of people with STIs (chlamydia, gonorrhoea, syphilis and trichomoniasis). It is estimated that there are 417 million prevalent cases of infection with the herpes virus (HSV-1/HSV-2) and approximately 291 million women infected with the human papillomavirus. ${ }^{16}$ Users of dating apps are generally young. Thirty-eight per cent are between 16 and 24 years old, and $45 \%$ are between 25 and 34 years old. Among the approximately 50 million active Tinder users, the number of men's profiles is $62 \%$ and the number of women's profiles is $38 \%$. Therefore, social media and dating apps increase communication and interaction between young people and adults at any age of sexual activity. ${ }^{17} 18$

Although social media and dating apps have worrying factors for the transmission of STI, they also offer possible intervention scenarios in that they can supply information about sexual health and risky behaviour, helping their users to engage in protected sexual activity. ${ }^{18}$ Currently, there is a shortage of research that explores this relationship and its potential impact. Traditional studies fail to elucidate a strong relationship between the use of websites and apps with increased risky sexual behaviour and STI. This is especially the case for women. Thus, in order to better understand this correlation, it is necessary, through a systematic review, to verify whether the use of websites and mobile apps favours the increase of risky sexual behaviours and susceptibility to an STI. ${ }^{19} 20$ 


\section{Objectives}

The purpose of this systematic review and meta-analysis is to assess the effect of the use of dating sites and apps by women on their level of risky sexual behaviours and incidence of STIs.

In addition, the goal is to assess the incidence of STIs/ HIV among women who use apps and whether these users have sex with a greater number of partners.

\section{METHODS AND ANALYSIS}

This protocol is registered in the International Prospective Register of Systematic Reviews (PROSPERO): CRD42019120494. The guidelines of the Preferred Reporting Items for Systematic Reviews and Meta-Analyses (PRISMA) were used to design this systematic review and meta-analysis protocol. $^{21}$

\section{Inclusion criteria}

Clinical trials and observational studies that reported on the use of social networks and dating apps and their associated effects on women's sexual behaviour and/or sexual health were evaluated. Inclusion criteria for studies are as follows: (1) focused on the use of websites and apps by women or involved app users and non-users; and (2) reported data on sexual behaviours, sexual health and STI. There will be no language restrictions when selecting studies.

\section{Patients, interventions, comparator/control and outcome \\ Patients: women who practice sexual relations. \\ Interventions: use of websites or mobile apps. \\ Comparator/control: non-use of websites or mobile apps.}

Outcome: increases in the risks of contracting a STI.

\section{Types of patients}

Sexually active women aged 18 years and over who do or do not use apps and social networking sites.

\section{Types of interventions}

Studies that show the use of dating sites or apps by women, such as Facebook, Instagram, Tinder, Badoo and others, to assess sexually risky behaviour and STI.

\section{Type of outcome measures}

Finding sexual partners through geosocial networks and dating apps enables users to have a greater number of sexual partners with increased turnover, consequently decreasing safe sexual practices and reducing transmission time of STIs. ${ }^{1822}$ The outcome examined will be the number of cases of STIs and AIDS among women using dating sites and apps. ${ }^{18}$

In a study conducted with users of apps for meeting sexual partners, it was reported by the interviewees that 42.6\% had recently been involved in group sex and $59.9 \%$ had anal intercourse without a condom. Group sex participation in the past 3 months were more common among those who reported positive HIV status $(65.2 \%)$ compared with those who reported negative $(41.4 \%)$ or unknown (10.0\%) HIV status. ${ }^{12}$

Applications can potentially promote risky behaviours because users can access them via cell phones at any time. The latter has been shown that app users are more likely to be diagnosed with STI than non-users; thus, they have a higher prevalence of risky sexual behaviours and are at more risk for STIs and HIV infection. ${ }^{7}$ Tang $e t a l^{23}$ found that $66.7 \%$ of homosexual app users did not ask about HIV status before meeting their most recent app partner in person. Evidence shows that advances in the use of apps and the increase in people using them to find sexual partners can have adverse effects on sexual health given the high prevalence of risky sexual behaviours.

\section{Patient and public involvement}

This is a protocol for a systematic review and meta-analysis that will be based on a wide-ranging and comprehensive bibliographic search of relevant databases. The individual data of the participants will not be included; thus, they will not be involved in defining the research terms, the determination of the measurement instruments, the implementation of the study design or the analysis of the data.

\section{Search strategy}

The Cochrane Central Controlled Trials Registry, ClinicalTrials.gov, MEDLINE, Embase, SciELO, Web of Science, Scopus and Cumulative Index to Nursing \& Allied Health Literature will be used to search for articles published between January 1990 and July 2020. We have selected publications starting in January 1990 because the first social network, Classmates.com, was created in $1990 .^{24}$

The Medical Subject Headings terms will be: Women AND (Mobile application OR Mobile apps OR Smartphone OR Online social networking OR Social networking OR Geosocial networking application) AND (Sexually transmitted infections OR STIs OR sexual behavior OR sexual health OR HIV) (table 1).

\section{Search strategy}

Table 1 presents the search strategy for MEDLINE.

\section{MEDLINE search strategy}

Eligible studies will also be selected from the reference lists of the retrieved articles.

\section{Data collection and analysis}

Selection of studies

Three authors, JFQ, KSM and MNM, will independently screen research results by using titles and abstracts. Duplicate studies and reviews will be excluded. Three reviewers, JFQ, ACAS and RNC, will go through the full text to determine whether the studies meet the inclusion criteria. Discrepancies will be resolved by a third reviewer, AKG. The study selection process is presented in a PRISMA flow chart $^{25}$ in figure 1. 


\section{Table 1 MEDLINE search strategy}

\section{Search items}

\begin{tabular}{|c|c|}
\hline 1 & Women \\
\hline 2 & Mobile application \\
\hline 3 & Mobile apps \\
\hline 4 & Smartphone \\
\hline 5 & Online social networking \\
\hline 6 & Social networking \\
\hline 7 & Geosocial networking application \\
\hline 8 & $\begin{array}{l}\text { Mobile application OR Mobile apps OR Smartphone } \\
\text { OR Online social networking OR Social networking OR } \\
\text { Geosocial networking application }\end{array}$ \\
\hline 9 & Sexually transmitted infections \\
\hline 10 & STIs \\
\hline 11 & Sexual behavior \\
\hline 12 & Sexual health \\
\hline 13 & HIV \\
\hline 14 & $\begin{array}{l}\text { Sexually transmitted infections OR STIs OR Sexual } \\
\text { behavior OR Sexual health OR HIV }\end{array}$ \\
\hline 15 & $\begin{array}{l}\text { Women AND (Mobile application OR Mobile apps OR } \\
\text { Smartphone OR Online social networking OR Social } \\
\text { networking OR Geosocial networking application) AND } \\
\text { (Sexually transmitted infections OR STIs OR Sexual } \\
\text { behavior OR Sexual health OR HIV) }\end{array}$ \\
\hline
\end{tabular}

\section{Data extraction and management}

Various characteristics of the eligible studies will be extracted, including the first authors' last names, year of publication, location of the study (country), study design, primary objective, population, sample size, follow-up period, inclusion/exclusion criteria, type of mobile app or sites used, type of control and STI testing results. Standardised data extraction forms will be specifically created

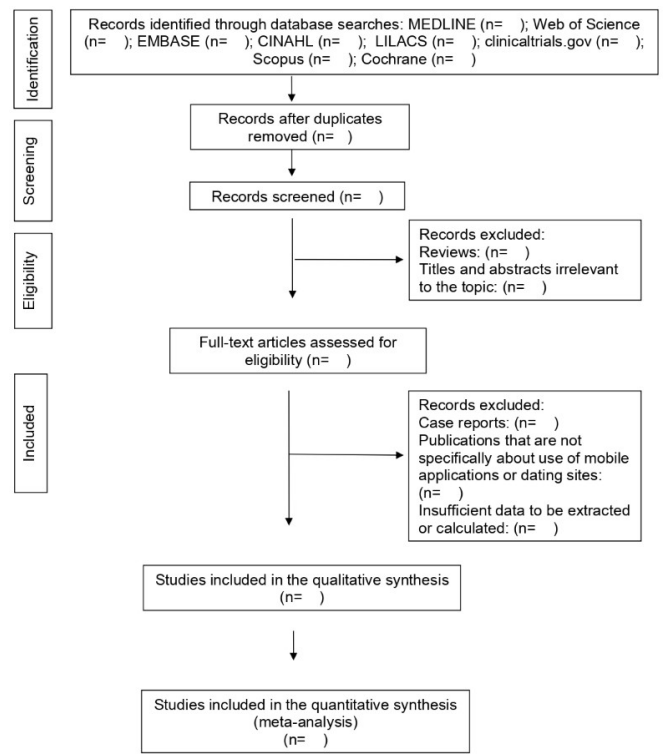

Figure 1 Flow diagram of the search for eligible studies in the use of mobile applications for dating site. for this review, and the results will be subsequently entered into a database. All data entries will be double-checked.

\section{Addressing missing data}

We will attempt to obtain any missing data by contacting the first or corresponding authors or coauthors of an article via phone, email or post. If we fail to receive any necessary information, the data will be excluded from our analysis and will be addressed in the discussion section.

\section{Risk-of-bias assessment}

Three authors, KSM, JFQ and BS, will independently assess the risk of bias in the eligible studies using the Cochrane risk of bias tool and Risk Of Bias in Non-randomized Studies of Interventions (ROBINS-I). ${ }^{25} 26$ The modified Cochrane Collaboration tool will be used to assess the risk of bias. Using the modified Cochrane Collaboration tool, individual elements from five domains (selection, performance, attrition, reporting and others) will be rated as high, low or unclear for each study. The risk of bias for non-randomised studies will be assessed using the ROBINS-I tool. This tool was designed to assess the risk of bias in reviews with questions related to interventions, aetiology, diagnosis and prognosis. ${ }^{26}$

\section{Assessment of heterogeneity}

The heterogeneity between study results will be evaluated using a standard $\chi^{2}$ test with a significance level of $\mathrm{p}<0.1$. To assess heterogeneity, we plan to compute the $\mathrm{I}^{2}$ statistic, which is a quantitative measurement of inconsistency across studies. If the level of heterogeneity is acceptable, we will perform a meta-analysis. If the data are heterogeneous, we will provide a narrative description of the findings.

\section{Analysis}

The data will be entered into Review Manager software (RevMan V.5.2.3, the Nordic Cochrane Center, Copenhagen, Denmark). The latter software allows the user to enter protocols, complete reviews, include text, characteristics of the studies, comparison tables, and study data and perform meta-analyses. For dichotomous outcomes, we will extract or calculate the OR and $95 \%$ CI for each study. In the case of heterogeneity $\left(\mathrm{I}^{2} \geq 50 \%\right)$, the randomeffects model will be used to combine the studies to calculate the OR and 95\% CI, using the DerSimonian-Laird algorithm in the metafor package (Wolfgang Viechtbauer, Maastricht, The Netherlands), which provides functions for conducting meta-analyses in $\mathrm{R}$ ( $\mathrm{R}$ Foundation for Statistical Computing, Vienna, Austria).

If the meta-analysis cannot be performed for all or some of the included studies, other characteristics and results of the study will be summarised narratively. In relation to the quality of the study and sample size, sensitivity analyses will be conducted to explore the robustness of the findings of studies included in the meta-analysis. Results of the sensitivity analyses will be presented in a summary table. 


\section{Grading quality of evidence}

We will use the appraisal rating, development and assessment approach to classify the strength of the evidence from the included data. The summary of the assessment will be incorporated into broader measurements to ensure the judgement of the risk of bias, consistency, openness and accuracy. ${ }^{27}$

\section{DISCUSSION}

The increasing use of dating sites and apps has expanded the number of potential sexual partners for the users of the technology, which has increased rates of sexual encounters. In this systematic review and meta-analysis, our goal is to consider the relationship between the use of dating sites and apps and risky sexual behaviours and incidence of STIs among women. ${ }^{18}$

Mobile apps facilitate greater communication between users. Through the use of this technology, women meet more people and have more opportunities for encounters, which can lead to risky sexual behaviour and can increase their likelihood of getting an STI. ${ }^{18}$

In a systematic review, Wang et al ${ }^{7}$ evaluated the existing evidence on the use of dating apps and associated sexual behaviours among app users, comparing the number of STIs between users and non-users of these apps. However, in the end, there were few eligible studies and the sample sizes were too small to perform the meta-analysis. However, other studies have shown that people who use dating apps are more likely to engage in risky sexual behaviours. 910

In contrast, in New York, the Teens in NYC app helped teenagers discover and access a wide range of sexual health services, including contraception. Another study showed an app for men that sent preventive notifications to users when a high probability of having risky sexual intercourse was detected, such as preventive and educational messages. Thus, these types of apps can become important tools for STI prevention. ${ }^{28} 29$

Thus, we hope that our study will shed light on the role of dating sites and mobile apps in the context of their effects on women's sexual expression and aid in improving access to sexual health information and reducing risky sexual behaviours among women.

\section{ETHICS AND DISSEMINATION}

Ethical approval was not required because this systematic review will use previously published data. The findings of this systematic review will be published in a peer-reviewed journal and will be updated if there is enough new evidence to change its conclusions.

\footnotetext{
Author affiliations

${ }^{1}$ Postgraduate Program in Health Sciences, Federal University of Rio Grande do Norte, Natal, Brazil

${ }^{2}$ Postgraduate Program in Woman Health, MEJC/EBSERH, Natal, Brazil

${ }^{3}$ Department of Biomedical Engineering, Federal University of Rio Grande do Norte, Natal, Brazil
}

${ }^{4}$ Department of Gynecology and Obstetrics, Federal University of Rio Grande do Norte, Natal, Brazil

Acknowledgements We would like to thank all coauthors. We would also like to extend our thanks to the China Health and Retirement Longitudinal Study team for providing data and the training of using the dataset.

Contributors JFQ, BS and AKG designed this systematic review and meta-analysis. JFQ drafted the manuscript, and AKG revised it. JFQ, RNC and AKG developed the search strategies, and JFQ, KSM, ACAS and MNM will implement it. JFQ, KSM, ACAS and MNM will conduct the literature search, extract the data and assess the quality of the included studies. In case of disagreement between the authors, AKG will advise on the methodology and will serve as the referee. RNC will complete the data synthesis. All authors have approved the final version of this manuscript.

Funding This paper was supported by the Beijing Municipal Natural Science Foundation (No. 9204025). The study sponsor has no role in study design, data analysis and interpretation of data, the writing of manuscript, or the decision to submit the paper for publication.

Competing interests None declared.

Patient and public involvement Patients and/or the public were not involved in the design, or conduct, or reporting, or dissemination plans of this research.

Patient consent for publication Not required.

Provenance and peer review Not commissioned; externally peer reviewed.

Open access This is an open access article distributed in accordance with the Creative Commons Attribution Non Commercial (CC BY-NC 4.0) license, which permits others to distribute, remix, adapt, build upon this work non-commercially, and license their derivative works on different terms, provided the original work is properly cited, appropriate credit is given, any changes made indicated, and the use is non-commercial. See: http://creativecommons.org/licenses/by-nc/4.0/.

ORCID iD

Ana Katherine Gonçalves http://orcid.org/0000-0002-8351-5119

\section{REFERENCES}

1 Blair SL, Claster PN, Claster SM. Technology and youth: growing up in a digital world. sociological studies of children and youth. Bingley: Emerald, 2015.

2 Czarny HN, Broaddus MR. Acceptability of HIV prevention information delivered through established geosocial networking mobile applications to men who have sex with men. AIDS Behav 2017;21:3122-8.

3 Chow JY, Klausner JD. Use of geosocial networking applications to reach men who have sex with men: progress and opportunities for improvement. Sex Transm Infect 2018;94:396-7.

4 Zeng EY, Vilardaga R, Heffner JL, et al. Predictors of utilization of a novel smoking cessation smartphone APP. Telemed $J$ E Health 2015;21:998-1004.

5 Holloway IW, Dunlap S, Del Pino HE, et al. Online social networking, sexual risk and protective behaviors: considerations for clinicians and researchers. Curr Addict Rep 2014;1:220-8.

6 Palmer JRB, Espenshade TJ, Bartumeus F, et al. New approaches to human mobility: using mobile phones for demographic research. Demography 2013;50:1105-28.

7 Wang H, Zhang L, Zhou Y, et al. The use of geosocial networking smartphone applications and the risk of sexually transmitted infections among men who have sex with men: a systematic review and meta-analysis. BMC Public Health 2018;18:1178.

8 Watchirs Smith L, Guy R, Degenhardt L, et al. Meeting sexual partners through Internet sites and smartphone Apps in Australia: national representative study. J Med Internet Res 2018;20:e10683.

9 Landovitz RJ, Tseng C-H, Weissman M, et al. Epidemiology, sexual risk behavior, and HIV prevention practices of men who have sex with men using GRINDR in Los Angeles, California. J Urban Health 2013;90:729-39.

10 Lehmiller JJ, loerger M. Social networking smartphone applications and sexual health outcomes among men who have sex with men. PLoS One 2014;9:e86603.

11 Chow JY, Konda KA, Calvo GM, et al. Demographics, behaviors, and sexual health characteristics of high risk men who have sex with men and transgender women who use social media to meet sex partners in Lima, Peru. Sex Transm Dis 2017;44:143-8. 
12 Goedel WC, Duncan DT. Correlates of engagement in group sex events among men who have sex with men in London who use geosocial-networking smartphone applications. Int J STD AIDS 2018;29:244-50.

13 Cheng SL, Rebecca G, José AB, et al. Trajectory of use over time of an oral tablet and a rectal gel for HIV prevention among transgender women and men who have sex with men. $J$ AIDS Care 2019;31:379-87.

14 Muessig KE, Pike EC, Legrand S, et al. Mobile phone applications for the care and prevention of HIV and other sexually transmitted diseases: a review. J Med Internet Res 2013;15:e1.

15 Alhassan RK, Abdul-Fatawu A, Adzimah-Yeboah B, et al. Determinants of use of mobile phones for sexually transmitted infections (STIs) education and prevention among adolescents and young adult population in Ghana: implications of public health policy and interventions design. Reprod Health 2019;16:120.

16 World Health Organization. Report on global sexually transmitted infection surveillance, 2018. Department of reproductive health and research World Health organization: 01-02, 2018.

17 Watchirs Smith L, Guy R, Degenhardt L, et al. Meeting sexual partners through Internet sites and smartphone Apps in Australia: national representative study. J Med Internet Res 2018;20:e10683.

18 Kesten JM, Dias K, Burns F, et al. Acceptability and potential impact of delivering sexual health promotion information through social media and dating apps to MSM in England: a qualitative study. BMC Public Health 2019;19:1236.

19 Shapiro GK, Tatar O, Sutton A, et al. Correlates of tinder use and risky sexual behaviors in young adults. Cyberpsychol Behav Soc Netw 2017;20:727-34.

20 Rebecca L, Collins CM, Rebecca S. Influence of new media on adolescent sexual health: evidence and opportunities. U.S. Department of Health \& Human Services 2011.
21 Moher D, Liberati A, Tetzlaff J, et al. Preferred reporting items for systematic reviews and meta-analyses: the PRISMA statement. PLoS Med 2009;6:e1000097.

22 Hoenigl M, Little SJ, Grelotti D, et al. GrindrTM users take more risks but are more open to HIV pre-exposure prophylaxis: could this dating APP provide platform for HIV prevention outreach? Clin Infect Dis 2019:ciz1093.

23 Tang W, Best J, Zhang Y, et al. Gay mobile apps and the evolving virtual risk environment: a cross-sectional online survey among men who have sex with men in China. Sex Transm Infect 2016;92:508-14.

24 Grov C, Breslow AS, Newcomb ME, et al. Gay and bisexual men's use of the Internet: research from the 1990s through 2013. J Sex Res 2014;51:390-409.

25 Higgins JPT, Altman DG, Gøtzsche PC, et al. The Cochrane Collaboration's tool for assessing risk of bias in randomised trials. BMJ 2011;343:d5928-9.

26 Brazil Ministry of Health. Secretariat of science, technology and strategic inputs. department of science and technology. ROBIS Risk of Bias in systematc reviews: tool to assess the risk of bias in systematic reviews: guidelines of use/Ministry of health, secretariat of science, technology and strategic inputs, department of science and technology. Brasília: Ministry of Health, 2017.

27 Balshem H, Helfand M, Schünemann HJ, et al. Grade guidelines: 3. rating the quality of evidence. J Clin Epidemiol 2011;64:401-6.

28 Steinberg A, Griffin-Tomas M, Abu-Odeh D, et al. Evaluation of a mobile phone APP for providing adolescents with sexual and reproductive health information, New York City, 2013-2016. Public Health Rep 2018;133:234-9.

29 Besoain F, Perez-Navarro A, Caylà JA, et al. Prevention of sexually transmitted infections using mobile devices and ubiquitous computing. Int J Health Geogr 2015;14:18. 